\title{
Multibuilding Block Janus Synthesized by Seed-Mediated Self-Assembly for Enhanced Photothermal Effects and Colored Brownian Motion in an Optical Trap
}

\author{
Kanokwan Sansanaphongpricha, Michael C. DeSantis, Hongwei Chen, Wei Cheng, \\ Kai Sun, Bo Wen, and Duxin Sun*
}

The asymmetrical features and unique properties of multibuilding block Janus nanostructures (JNSs) provide superior functions for biomedical applications. However, their production process is very challenging. This problem has hampered the progress of JNS research and the exploration of their applications. In this study, an asymmetrical multibuilding block gold/iron oxide JNS has been generated to enhance photothermal effects and display colored Brownian motion in an optical trap. JNS is formed by seed-mediated self-assembly of nanoparticle-loaded thermocleavable micelles, where the hydrophobic backbones of the polymer are disrupted at high temperatures, resulting in secondary self-assembly and structural rearrangement. The JNS significantly enhances photothermal effects compared to their homogeneous counterpart after near-infrared (NIR) light irradiation. The asymmetrical distribution of gold and iron oxide within JNS also generates uneven thermophoretic force to display active colored Brownian rotational motion in a single-beam gradient optical trap. These properties indicate that the asymmetrical JNS could be employed as a strong photothermal therapy mediator and a fuel-free nanoscale Janus motor under NIR light.

\section{Introduction}

Janus, anisotropic, or patchy particles have long been in an interest of various applications. Their asymmetrical structures make them appealing for diverse functions, such as surface-reducing agents, electronic devices, catalysts, molecular imaging, diagnostics, and drug delivery systems. ${ }^{[1-4]}$ Recently,

K. Sansanaphongpricha, Dr. M. C. DeSantis, Dr. H. Chen, Prof. W. Cheng, Dr. B. Wen, Prof. D. Sun Department of Pharmaceutical Sciences University of Michigan Ann Arbor, MI 48109, USA

E-mail: duxins@umich.edu

Dr. K. Sun

Department of Material Sciences and Engineering University of Michigan

Ann Arbor, MI 48109, USA

DOI: $10.1002 / \mathrm{smll} .201602569$ multibuilding block Janus, composed of multiple nanoparticles arranged in an asymmetrical pattern in a single entity, has been used in imaging, theranostics, and cargo deliveries. ${ }^{[5-7]}$ These complex Janus structures provide superior properties and have more effective responses to external stimuli compared to a single-domain Janus particle of the same size. The multibuilding block Janus benefits from the high surface-tovolume ratio $^{[8]}$ and strong interaction among metal nanoparticle building blocks. ${ }^{[9]}$ Despite the exceptional qualities of the multibuilding block Janus particles, the approach and control process to produce these complex structures on a nanoscale are very challenging. ${ }^{[10]}$ They require a high degree of control to direct the aggregate process. Conventional approaches for Janus nanoparticle (JP) production, such as surface masking and phase separation, mostly yield single domain JPs on a micrometer scale, which have limited use for biomedical applications. ${ }^{[11-14]}$ Those strategies are not suitable for fabricating nanoscale multibuilding block JPs. The most promising strategy for multibuilding block 
JP formation on a nanometer scale is to employ bottom up self-assembly to control the location of nanoparticle distribution in block copolymers. Previous research has suggested that many factors significantly contribute to the location of nanoparticles inside micelles, such as affinities between nanoparticle-coating ligands and polymeric micelles, ${ }^{[15]}$ relative lengths of polymer and nanoparticle size, ${ }^{[16,17]}$ lengths of surface coating of nanoparticles, ${ }^{[16]}$ glass transition temperature of the polymer, ${ }^{[18]}$ and solvent effects. ${ }^{[19,20]}$ These significant discoveries allow researchers to control the location of nanoparticles and form complex structures such as nanowires, nanosheets, cylinders, and vesicles. ${ }^{[21]}$ However, not much research has focused on utilizing controlled nanoparticle location and self-assembly to fabricate a nanoscale asymmetrical multibuilding block Janus structure composed of two different inorganic nanoparticles.

Gold (AuNP) and iron oxide (IONP) nanoparticles are the most intensively studied inorganic nanoparticles. They show good safety profiles, biocompatibility, biodegradability, and efficacy in cancer theranostics. ${ }^{[22,23]}$ Most importantly, AuNPs and IONPs have different photothermal conversion efficiencies. The temperature created by AuNP and IONP are different. As a result, the gold/iron oxide Janus nanostructure (JNS) experiences a thermophoretic force that causes the particle to rotate. Jiang et al. have shown that a micrometersized gold/silica Janus can generate a spin motion under $1064 \mathrm{~nm}$ laser irradiation due to self-thermophoresis of the microparticles, which can be visualized under microscope. ${ }^{[24]}$ However, it is challenging to generate active motion in nanosized Janus structures, and even more difficult to visualize that active rotational motion. While optical trapping of JNS has been previously demonstrated, those experiments were not designed to detect the active motion. ${ }^{[25]}$ Therefore, we intend to create an active spin motion from a nanoscale Janus, and use a modified optical trapping technique to detect the unique motion. An asymmetrical Janus structure has the potential to generate active motion for electrical and biomedical applications. ${ }^{[24]}$

In this article, we report a novel fabrication of multibuilding block Janus structures composed of two different inorganic nanoparticles, AuNP and IONP, using thermocleavable amphiphilic diblock copolymer to control the nanoparticle distribution and self-assembly of nanoparticles loaded in the thermocleavable micelles (TCMs). To the best of our knowledge, this is, for the first time, using thermocleavable polymer to form asymmetrical Janus structures. The thermocleavable amphiphilic diblock copolymer, in which the hydrophobic backbone could be cleaved apart at high temperatures via retro Diels-Alder (DA) reaction, provides an unstable condition at high temperatures, which encourages AuNPs and IONPs to self-assemble into multibuilding block JNS by micelle collision and fusion. We found that our JNS expressed strong photothermal efficiency after near-infrared (NIR) light irradiation, much stronger than that of their homogeneous counterparts, due to the strong collective effect of their AuNPs. The NIR laser light irradiation also created a temperature imbalance between the two different sides of the JNS, which caused the particles to rotate, resulting in distinct frequencies of colored Brownian motion under an optical trap. These unique characteristic suggest that the $\mathrm{Au} /$ IONP JNS could potentially be employed as for both photothermal therapy (PTT) and nanoscale Janus motors at the same time. ${ }^{[26]}$ This is highly significant for the future research in vivo, where the penetration of nanoparticles in a tumor mass is limited at only the shallow layers of the tumor around blood vessels. Our JNS can possibly overcome this problem as they can eradicate the cancer cells near the blood vessels and also simultaneously penetrate to the deeper layers of the tumor mass by self-propelled motor and mechanical sheer force. ${ }^{[27-29]}$

\section{Results and Discussion}

\subsection{Thermocleavable Polymer Synthesis}

The JNSs were fabricated via self-assembly of nanoparticles loaded into thermocleavable polymeric micelles. We first synthesized the thermocleavable hydrophobic backbone via DA reaction at $70{ }^{\circ} \mathrm{C} \cdot{ }^{[30,31]}$ Molecular weight of the hydrophobic backbone is 5090 Da measured by gel permeation chromatography (GPC) and then poly ethylene oxide (PEO) molecular weight $5000 \mathrm{Da}$ was added and reacted with the maleimide functional group of the hydrophobic backbone via Michael addition. The final amphiphilic diblock thermocleavable polymer (Da- $b$-PEO) has the molecular weight of 9800 Da characterized by GPC and ${ }^{1} \mathrm{H}$ NMR (Figure 1a, Figures S1-S3 in the Supporting Information). This polymer acts not only as a key component to control hydrophobic interaction between nanoparticles and the polymer itself, but also mediates the micelle fusion. The cycloadducts in the hydrophobic backbones can be cleaved apart at or above $90{ }^{\circ} \mathrm{C}$ via retro DA reaction, ${ }^{[32]}$ as proven by ${ }^{1} \mathrm{H}$ NMR (Figure $1 \mathrm{~b}, \mathrm{c}$ ). The data suggest that $84 \%$ of the formed cycloadducts were cleaved after the polymer was treated at $100{ }^{\circ} \mathrm{C}$ for an hour (Figure 1d). The retro DA causes hydrophobic backbone shortening and hydrophobichydrophilic imbalance, which critically affects the thermodynamic stability and triggers self-assembly of the TCMs.

\subsection{Multibuilding Block Gold/Iron Oxide JNS Formation}

Gold and iron oxide nanoparticles were used to generate JNSs. Oleic acid-capped iron oxide nanoparticles $(15 \mathrm{~nm})$ (IONPs) and dodecanethiol capped gold nanoparticles $(5 \mathrm{~nm})$ (AuNPs) were encapsulated separately in the polymer to make IONP-loaded TCMs (FeTCMs) and AuNPloaded TCMs (AuTCMs), respectively. Transmission electron microscopy (TEM) images clearly showed that nanoparticles aggregated at the center of the micelles' hydrophobic domain (Figure 2a,b) due to strong interparticle Van Der Waals attractions. TEM data suggest that FeTCMs and AuTCMs have average core diameters of $39.6 \mathrm{~nm}$ and $56.6 \mathrm{~nm}$ respectively (Figure S4, Supporting Information). The hydrodynamic diameters of these two micelles are $78.82 \mathrm{~nm}$ and $94.88 \mathrm{~nm}$, which are larger than the core diameters measured by TEM because of the interaction between polymer and 
a

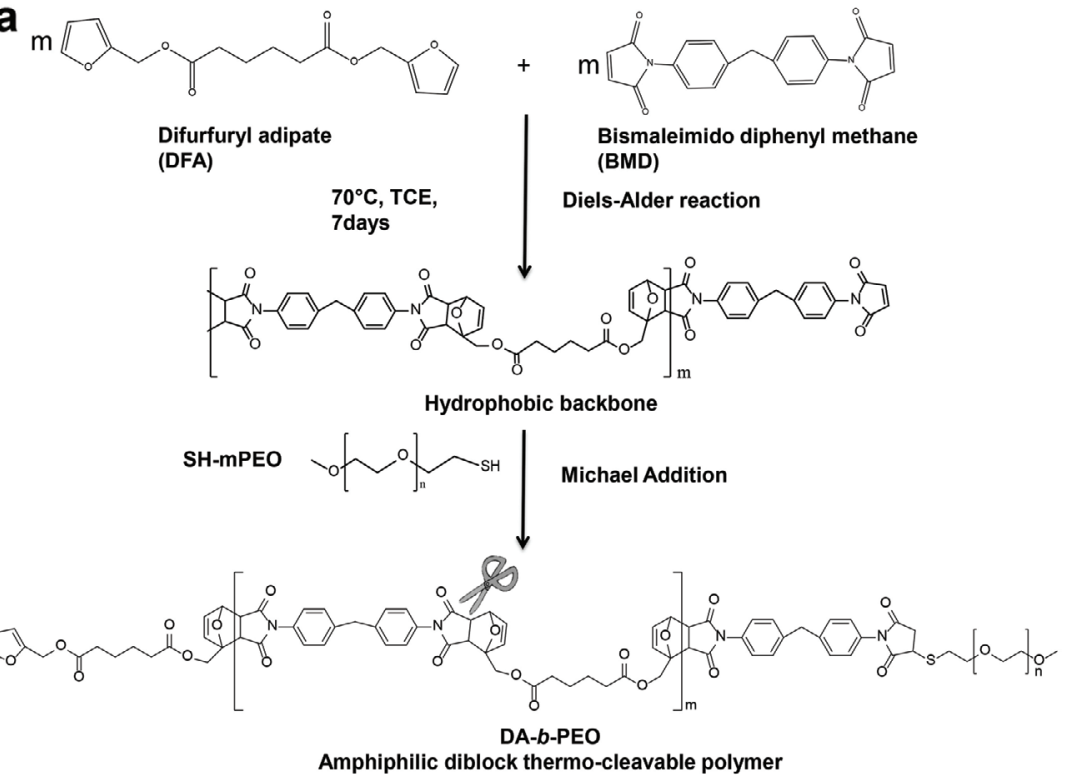

b



d

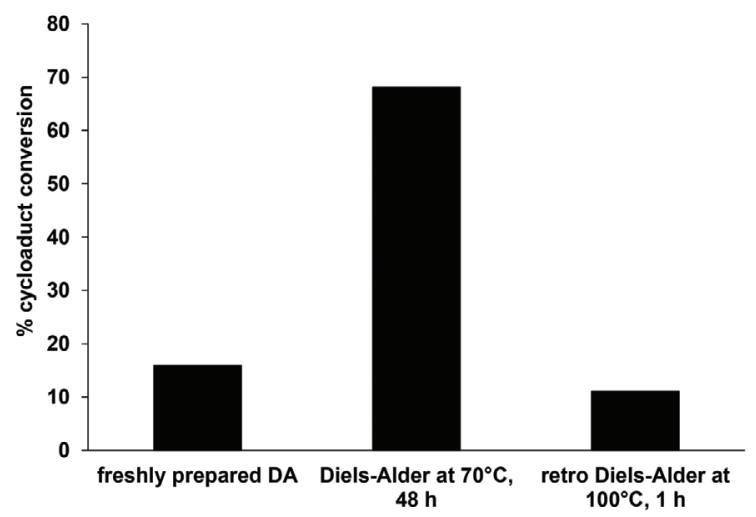

Figure 1. a) A synthesis scheme of DA- $b$-PEO amphiphilic diblock thermocleavable copolymer. An equimolar of DFA and BMD was mixed in tetrachloro ethane and the reaction was carried out at $70^{\circ} \mathrm{C}$ for $7 \mathrm{~d}$. The molecular weight of the polymer was $5090 \mathrm{Da}$. Then SH-mPEG was conjugated with the maleimide terminus of the hydrophobic backbone via Michael addition and yielded the final product with the molecular weight of $9800 \mathrm{Da}$. b) A cartoon picture representing the thermocleavable polymer and the hydrophobic backbone cleavage after high temperature exposure. c) ${ }^{1} \mathrm{H}$ NMR of the hydrophobic backbone at different time points the aqueous media (Table S1, Supporting Information). Next, the excess molar concentrations of AuTCM were mixed with FeTCM, subsequently mixed together with the free TCM seeds. This was followed by heat treatment $\left(94^{\circ} \mathrm{C}\right)$ to generate asymmetrical Au/IONP JNS. The excess molar concentration of AuTCM was used to ensure that all FeTCMs were fused with AuTCMs. This completed solution was purified by a magnetic separator to remove the unreacted AuTCMs. The amount of AuNPs and IONPs in JNS could be controlled by the concentration ratios between AuTCMs and FeTCMs (Figure S5, Supporting Information). Percent yield of JNS is $97.16 \%$ calculated from the feeding amount of Fe in FeTCM. Transmission electron microcopy (TEM) (Figure 2c,d, and Figure S6a in the Supporting Information) and scanning transmission electron microscope high angle annular dark-field (STEM-HAADF) (Figure 2e, and Figure S6b in the Supporting Information) images illustrate that AuNPs and IONPs are combined together in a new single entity with a welldefined asymmetrical nanostructure. The average core diameter of the JNS measured by TEM is $86.5 \mathrm{~nm}$ (Figure S4, Supporting Information) and hydrodynamic diameter measured by dynamic light scattering (DLS) is $100.3 \mathrm{~nm}$. It is important to note that JNS formed by the selfassembly approach have a relatively small size, compared to Janus particles made by other conventional methods, which mostly yield particles in micrometer-scale single domains. ${ }^{[33]}$ We performed X-ray energy dispersive spectroscopy (XEDS) element mapping to confirm that the JNS are composed of multiple AuNPs and IONPs in an asymmetrical pattern (Figure 2f). The formed JNSs are stable over a period of 6 months after being stored at $4{ }^{\circ} \mathrm{C}$. DLS

and temperatures: freshly prepared (top), $48 \mathrm{~h}$ after $70^{\circ} \mathrm{C}$ heat treatment (middle), and $1 \mathrm{~h}$ after $100{ }^{\circ} \mathrm{C}$ heat treatment. It clearly shows that the cycloadducts peak at 3.09 and $5.32 \mathrm{ppm}$ increase after polymerization via Diels-Alder reaction at $70{ }^{\circ} \mathrm{C}$ for $48 \mathrm{~h}$. However, these peaks disappear after the temperature increases to $100{ }^{\circ} \mathrm{C}$ for an hour. This indicates the cycloadduct disruption via retro Diels-Alder. d) $84 \%$ of the cycloadducts were cleaved after being treated at $100{ }^{\circ} \mathrm{C}$ for an hour. The data suggest that the backbone cleavage is very efficient after $1 \mathrm{~h}$ of high temperature exposure. 


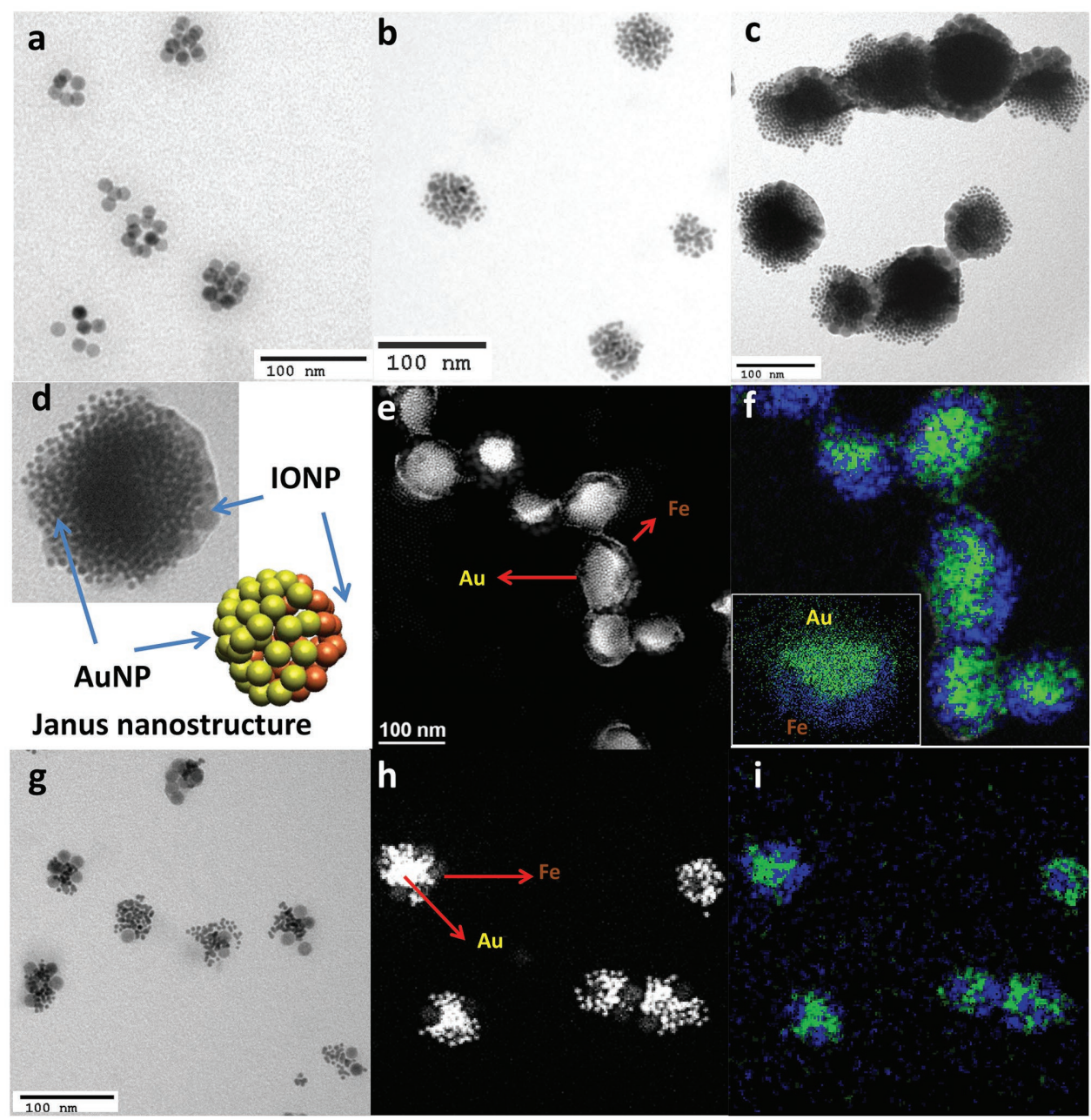

Figure 2. TEM images of Janus nanostructures (JNSs). a) The TEM images of the original FeTCM and b) AuTCM before heat treatment. c) A TEM image of multibuilding block Au/IONP JNS after self-assembly process. d) A high magnification TEM image and a cartoon picture show an asymmetrical structure of JNS. e) A STEM-HADDF image of JNS and f) XEDS element maps of JNS confirm an asymmetrical pattern of JNS. g) TEM, h) STEM, and i) XEDS images of GMC show a random pattern of Au/IONP mixture in micelles.

data indicate no large aggregation in the solution (Figure S7, Supporting Information). To prove that the thermocleavable polymer is a critical factor in the formation of our JNS, we used polystyrene- $b$-polyethylene oxide (PS- $b$-PEO, $\left.M_{\mathrm{w}} 10300 \mathrm{Da}\right)$ as a control for non-TCMs to encapsulate $15 \mathrm{~nm}$ IONPs and $5 \mathrm{~nm}$ AuNPs separately. This polymer cannot trigger self-assembly to form JNS after adding PS$b$-PEO seeds at high temperature. These two types of nanoparticle-loaded micelles remained separate (Figure S8, Supporting Information). We further ruled out the possibility that different capping ligands of AuNPs and IONPs cause a nonspecific asymmetrical structure inside of TCM in an independent manner of polymer properties and self-assembly process. First, we mixed AuNPs and IONPs together homogeneously, then loaded them into the thermocleavable polymer to form micelles. TEM, STEM-HAADF imaging, and XEDS element mapping show that no well-organized asymmetrical JNS formation occurred (Figure $2 \mathrm{~g}-\mathrm{i}$ ). Under these conditions, AuNPs and IONPs mixed together in micelles randomly, forming a homogeneous gold/iron oxide micelle counterpart (GMC) without any specific pattern. These data suggest that the thermocleavable polymer is critical for the self-assembly and self-reorganization processes that form our asymmetrical JNS.

\subsection{Ball-Like and Patchy Secondary Nanostructure Formation}

We further studied seed-mediated self-assembly by following similar procedures to those for JNS formation, but using only one species of metal nanoparticles, either iron oxide or gold, mixed with the TCM seeds. TEM images clearly show the selfassembly process forming iron oxide ball-like nanostructures (FeBL) with diameter $\approx 74.1 \mathrm{~nm}$ (Figure 3a, and Figure S4 in the Supporting Information). This transformed structure was also confirmed by STEM-HAADF (Figure 3b,c). The images and density profiles of FeBL (Figure 3d) clearly indicate that the electron density was higher at the edges and lower inside the cores because nanoparticles aligned at the interface between hydrophilic PEG and hydrophobic residues after self-assembly. Notably, the self-assembly and transformation of nanoparticle-loaded TCM occurs regardless of 


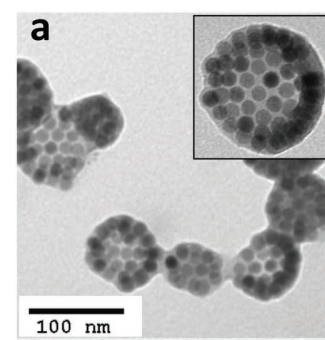

e

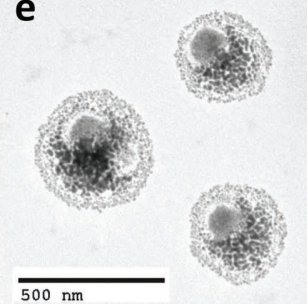

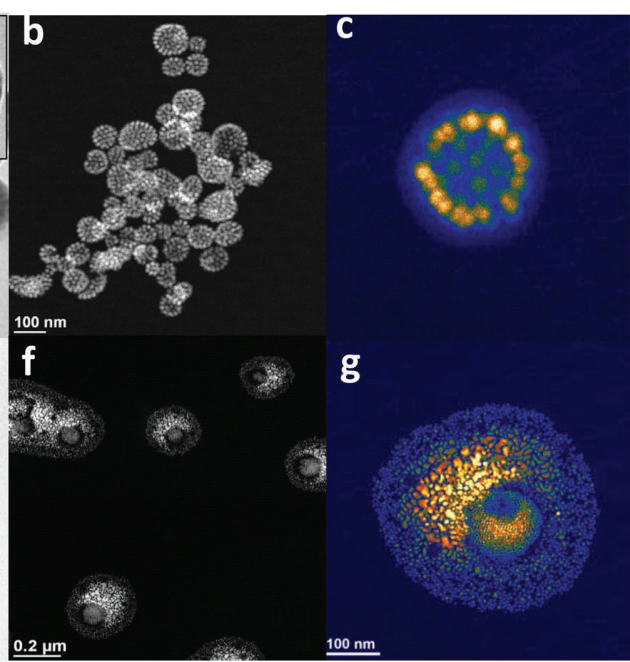

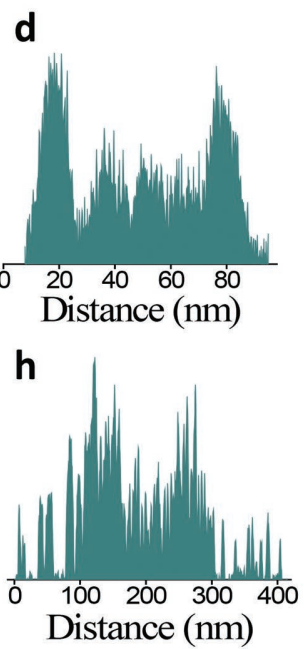

Figure 3. The TEM images of secondary structures. a,e) TEM images of FeBL and AuPS after self-assembly of FeTCM and AuTCM respectively. b,f) STEM-HADDF images of FeBL and AuPS at low magnifications. c,g) STEM-HADDF images of FeBL and AuPS at high magnifications with a color heat map. $d, h)$ Density profiles of FeBL and AuPS, implying that nanoparticles align at the polymer interface rather than inside the core.

nanoparticle type. We observed that self-assembly and transformation also took place with AuTCM. TEM and STEMHAADF images clearly show gold patchy nanostructures (AuPS) (Figure $3 \mathrm{e}-\mathrm{g}$ ). The density profile also confirmed an internal void volume of AuPS with a larger diameter than FeBL, $281.0 \mathrm{~nm}$ (Figure 3h). These data suggest that the selfassembly and self-reorganization process has transformed cluster nanoparticle micelles into secondary nanostructures. It is important to note that we did not observe self-reorganization and transformation using the IONP-loaded in PS- $b$-PEO micelles with the nonthermocleavable polymer (PS- $b$-PEO) seed (Figure S9, Supporting Information).

\subsection{Seed-Mediated Self-Assembly Process}

We further investigated the mechanism of JNS and secondary structure formation. It is clear that self-assembly and transformation are driven by thermodynamic force. Luo and Eisenberg previously demonstrated that an increased amount of an aqueous phase in micelle system could induce thermodynamic instability, leading to micelle fusion. Smaller micelles fused together and formed larger micelles in order to mitigate their interfacial energy penalty. ${ }^{[34,35]}$ Nevertheless, micelle fusion is quite unlikely in the case of nanoparticleloaded micelles. The fusion of micelles is highly dependent on the polymer's properties and the nanoparticle diameters. ${ }^{[36,37]}$ These NP-TCMs are stable, because the hydrophobic nanoparticles have a strong hydrophobic interaction within the hydrophobic polymer backbone. They are very rigid and less flexible, which makes micelle fusion and self-assembly of these NP-TCMs unusual. The hydrophobic attraction between the polymer backbone itself and the nanoparticles needs to be diminished in order to create an unstable state and to initiate the self-assembly process for micelle fusion of these FeTCMs and AuTCMs. We examined the mechanism of nanoparticle self-assembly by using only one species of metal nanoparticles and the free TCM seeds to reduce complexity. A series of TEM images confirms that AuTCM fused together until they were stable, then underwent phase separation and structural rearrangement. Samples for TEM were taken at the beginning of heat treatment (Figure 4a) and $2 \mathrm{~h}$ after the heat treatment began (Figure 4b-e). When the mixture of NPTCMs and free TCM seeds in aqueous media was exposed to the high temperature $\left(94^{\circ} \mathrm{C}\right)$, the hydrophobic backbones in all micelle species subsequently cleaved apart via retro DA reaction, resulting in a reduction of the hydrophobic attraction between the backbone and nanoparticles. At this stage, NP-TCMs become unstable and relatively flexible. As a consequence, these unstable NP-TCMs fused with free TCM seeds in the aqueous media. While one NP-TCM collides with the seed, a second NP-TCM can also fuse with the same seed from the opposite side (Figure 4f). The free TCM not only acts as a seed to mediate the self-assembly, but also enhances a depletion force between two NP-TCMs. ${ }^{[38,39]}$ This leads to structural transformation and self-assembly, forming the JNS. If only one species of NP-TCM (either AuTCMs or FeTCMs) is mixed with free TCM seeds in the system, patchy or balllike nanostructures will be formed instead of JNS. Although the self-assembly process is challenging to control, we were able to regulate JNS formation by varying the ratio between AuNPs and IONPs. In contrast, the system without free TCM seeds failed to transform into secondary nanostructures at a high temperature. It was observed that the hydrophobic nanoparticles were released into the aqueous media, where they precipitated (Figure S10, Supporting Information). Without free TCM seeds, there is neither a template for NP-TCMs to anchor onto nor a depletant to attract NP-TCMs; consequently, the fusion process is not possible.

The hydrophobic chain's changes in length under high temperature conditions are critical for JNS formation via the self-assembly process. It has been reported that the relation between lengths of polymeric micelles and nanoparticle diameters is a significant factor for controlling the location 

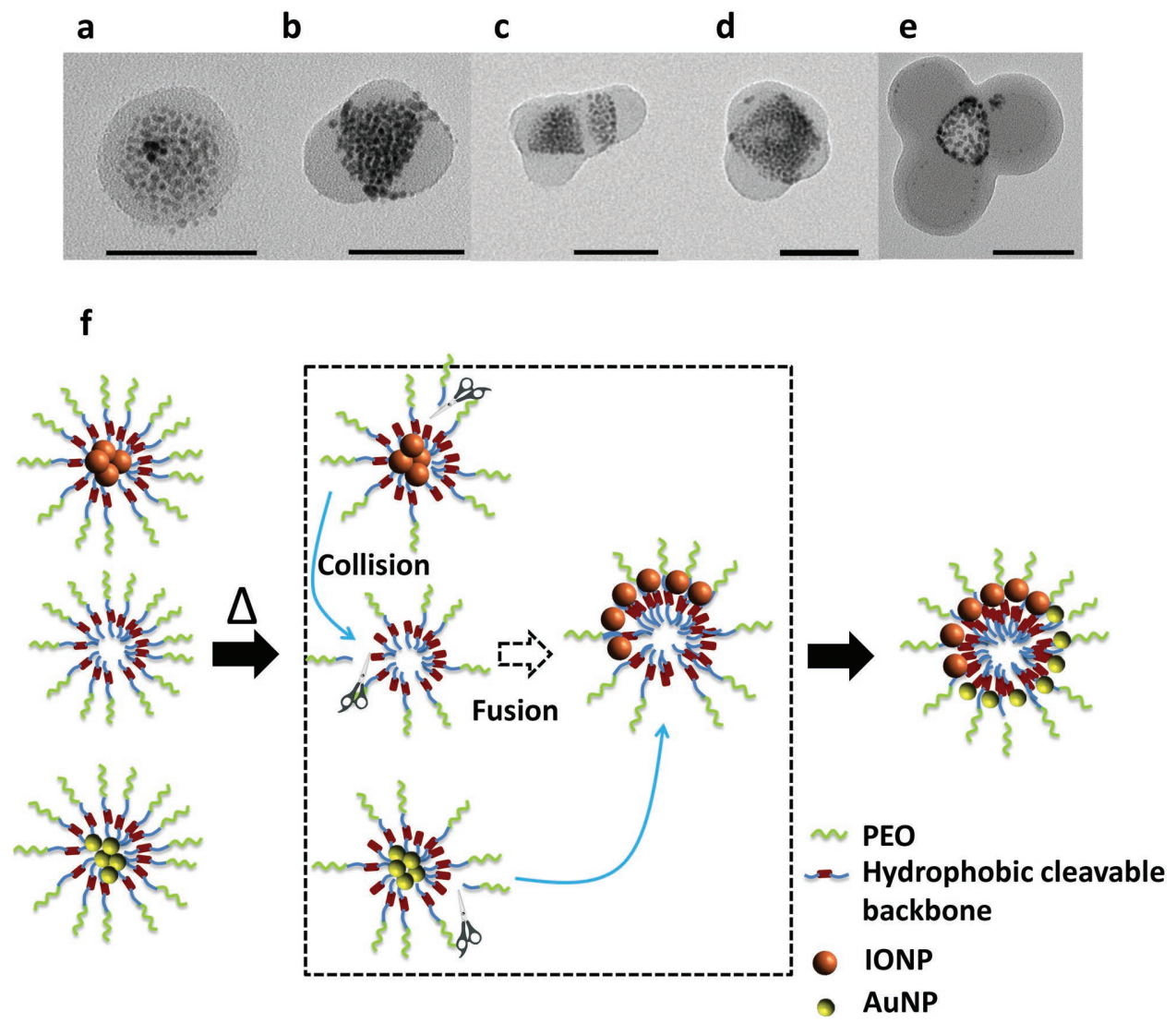

Figure 4. TEM images and scheme of self-assembly process. a) TEM image of AuTCM at high magnification. The sample was taken at the beginning of heat treatment. $b-d)$ Series of TEM images showing multiple AuTCMs fusion process. e) TEM image demonstrating internal phase separation and structural rearrangement to form secondary structures. Scale bars from panels (a-e) are $100 \mathrm{~nm}$. f) Schematic diagram demonstrates the transformation mechanism from cluster micelles to multibuilding block Janus or ball-like nanostructures. First, an FeTCM collides with free TCM seed. Simultaneously, another AuTCM can also collide with the same seed from the opposite direction and subsequently fuse together, resulting in self-reorganization to form JNS. If only one kind of NP-TCMs is used, ball-like or patchy structures will be formed instead of JNS.

of nanoparticles inside micelles. ${ }^{[16,17]}$ In general, the energy penalty increases with the increasing ratio between nanoparticle size and the coil dimension of the polymer. Since the nanoparticles' radius of gyration is larger than the polymer's radius of gyration after backbone cleavage, the nanoparticles are expelled from the matrix, and large-scale phase separation occurs. ${ }^{[40-42]}$

\subsection{JNS Enhances Photothermal Effect}

We further explored how our multibuilding block JNSs interact with photothermal effect. Intriguingly, Au/IONP JNS significantly enhance temperatures when undergoing $885 \mathrm{~nm}$ NIR laser light irradiation at low laser power, $0.45 \mathrm{~W}$, with low concentrations of iron $(40 \mu \mathrm{g} \mathrm{mL}-1)$ and gold $\left(170 \mu \mathrm{g} \mathrm{mL}^{-1}\right)$. Upon $10 \mathrm{~min}$ of NIR light irradiation, JNS gradually increased the temperature by $20.9{ }^{\circ} \mathrm{C}$ from the initial temperature in the cell culture media, while other controls, such as their homogeneous gold/iron oxide micelle counterparts (GMC), gold cluster micelles (AuTCM), iron oxide cluster micelles (FeTCM), a solution mixture of gold micelle and iron oxide cluster micelles, and cell culture media alone, increased only $15.0,12.7,7.3,13.4$, and $6.1{ }^{\circ} \mathrm{C}$ from the initial temperature, respectively (Figure 5a). Concentrations of gold and/or iron in all samples were fixed to the same or close to their concentration in the JNS. In addition, we further tested the enhanced photothermal effect on cancer cell. We monitored cell viability using an Alamar Blue assay when triple negative breast cancer cells (SUM159 cells) were treated with JNS under $885 \mathrm{~nm}$ NIR laser light. The high temperature created by the JNS resulted in lower cell viability compared to other treatments and the media control group. The cell viability of the group treated with JNS under NIR laser light irradiation was $62.76 \%$, while treatment using only the JNS, without NIR laser light irradiation, displayed no toxicity, with cell viability of $92.74 \%$ (Figure $5 b$ ).

In order to induce cell death by PTT, the temperature must be higher than $41{ }^{\circ} \mathrm{C}$ to unfold proteins and trigger cell death signaling. ${ }^{[43,44]}$ Therefore, good photothermal contrast agents with high photo-conversion efficiency are necessary. These agents must be able to significantly increase the temperature of the surrounding environment under low NIR laser power. ${ }^{[45,46]}$ This is very important for ideal in vivo PTT, since low laser power has a minimal effect on surrounding normal tissues but effectively kills the cancer cells. It is noteworthy that the surface of JNS is covered by hydrophilic PEO polymer. Though the hydrophobic backbone cleaves, 

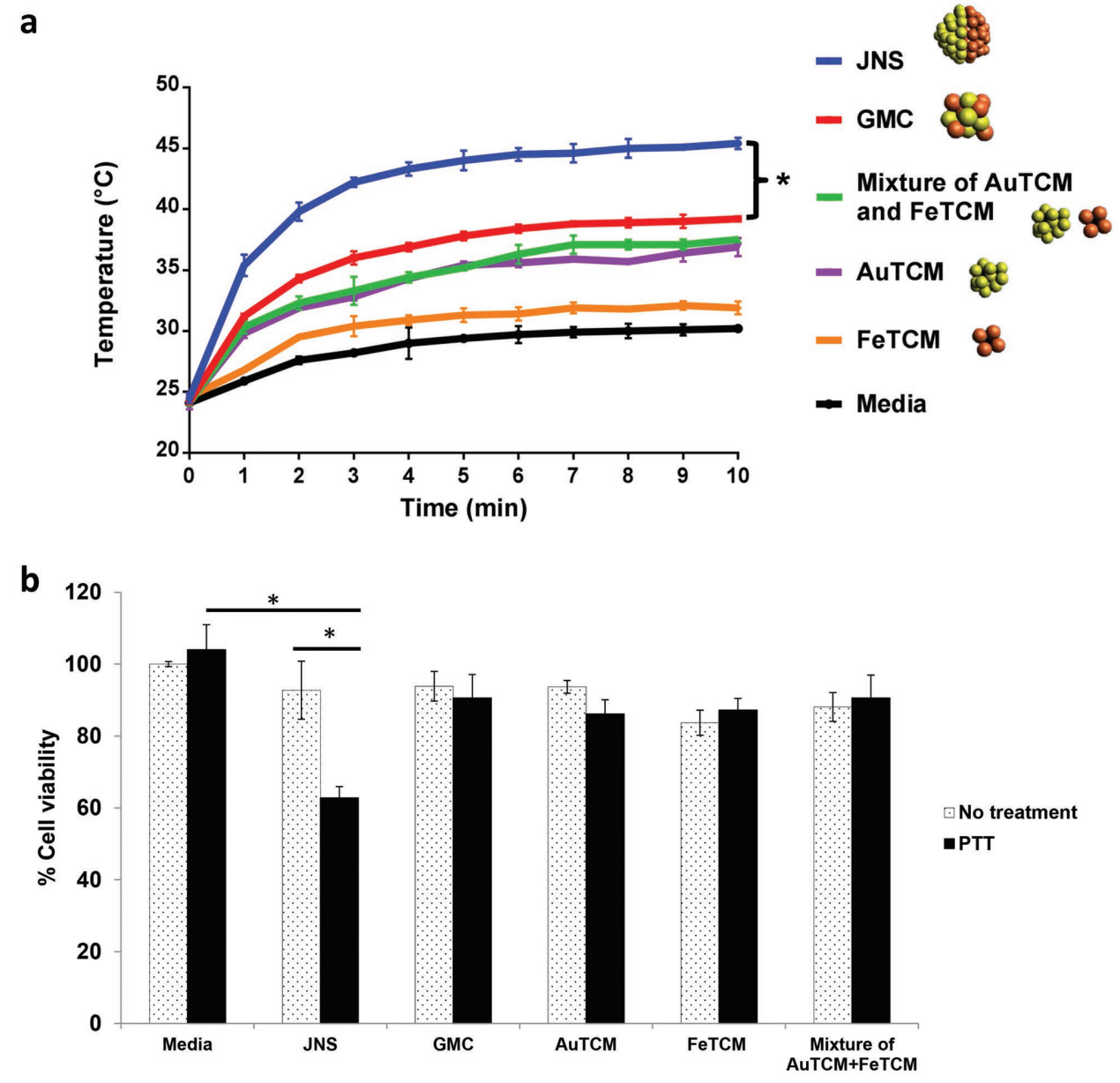

Figure 5. JNS enhances photothermal effect. a) Graph shows photothermal effect of different types of nanoparticles. JNS significantly enhances the temperature after NIR laser light irradiation at $885 \mathrm{~nm}$ for $10 \mathrm{~min}$ comparing to other structures. The asterisk shows significant difference, $P$-value $<0.05$. b) Percentage of SUM-159 cell viability with and without photothermal treatment in different types of nanoparticles. The asterisks show $P$-value $<0.05$.

the PEO remains intact. This makes JNS become stealthy and prevents protein absorption on the surface of JNS resulting in the reduction of phagocytosis by macrophages in vivo. Notably, our JNS demonstrates high photothermal efficiency at low laser power compared to its homogeneous counterparts. JNS clearly demonstrates overall higher absorption from 400-900 nm (Figure S11, Supporting Information). JNS may have "collective effects" by presence of multiple AuNPs in close proximity for higher PTT as described in the literature. ${ }^{[47]}$ Specifically, there are two mechanisms of interactions between AuNPs for the heating process: (1) accumulative effect of AuNP confined in JNS. The accumulative effect in AuNPs has been reported by Gorotov et al., resulting in the strong temperature enhancement. ${ }^{[48]}$ This effect stems from the addition of heat fluxes of multiple AuNPs. Our JNS shows absorption at $\lambda_{\max } 1.61$-fold stronger than the GMC at the same concentration of $\mathrm{Au}$ and Fe. (2). The Coulomb effect is generated from the interaction among AuNPs in the JNS, which is also supported by literature reported by Zhang et al. ${ }^{[49]}$ As a result, we observed a redshift from 504 to $520 \mathrm{~nm}$ of JNS compared to GMC. The insertion of IONPs between AuNPs in GMC hinders the accumulative and the Coulomb effect of AuNPs. Therefore, GMC could not fully take advantage of the collective effect. These data suggest that the structural arrangement of nanoparticles plays a crucial role in photothermal effect of secondary structures.

\subsection{JNSs Display Colored Brownian Motions under a Single-Beam Gradient Optical Trap}

Since our JNS's structure is asymmetrical, with AuNPs on one side and IONPs the other, the two sides have different photothermal efficiencies as well. ${ }^{[22,23]}$ The absorption of laser light by AuNPs and IONPs on the surface of the JNS creates an uneven temperature gradient. Therefore, the JNS experiences a thermophoretic force that causes the structure to rotate (Figure 6a). To validate this behavior and quantitate the rotation frequency, we employed an optical trap to monitor individual JNS for this active rotational motion at the focus of an NIR laser $(\lambda=830 \mathrm{~nm})$. Incorporating back-focal-plane interferometry (BFPI), this technique permits ultrasensitive tracking and characterization of trapped sub-micrometer particles as recently applied to HIV-1 virions in culture media. [50]

Power spectra from individually trapped particles are analyzed such that the laser alone is used as a baseline control 
$\mathbf{a}$
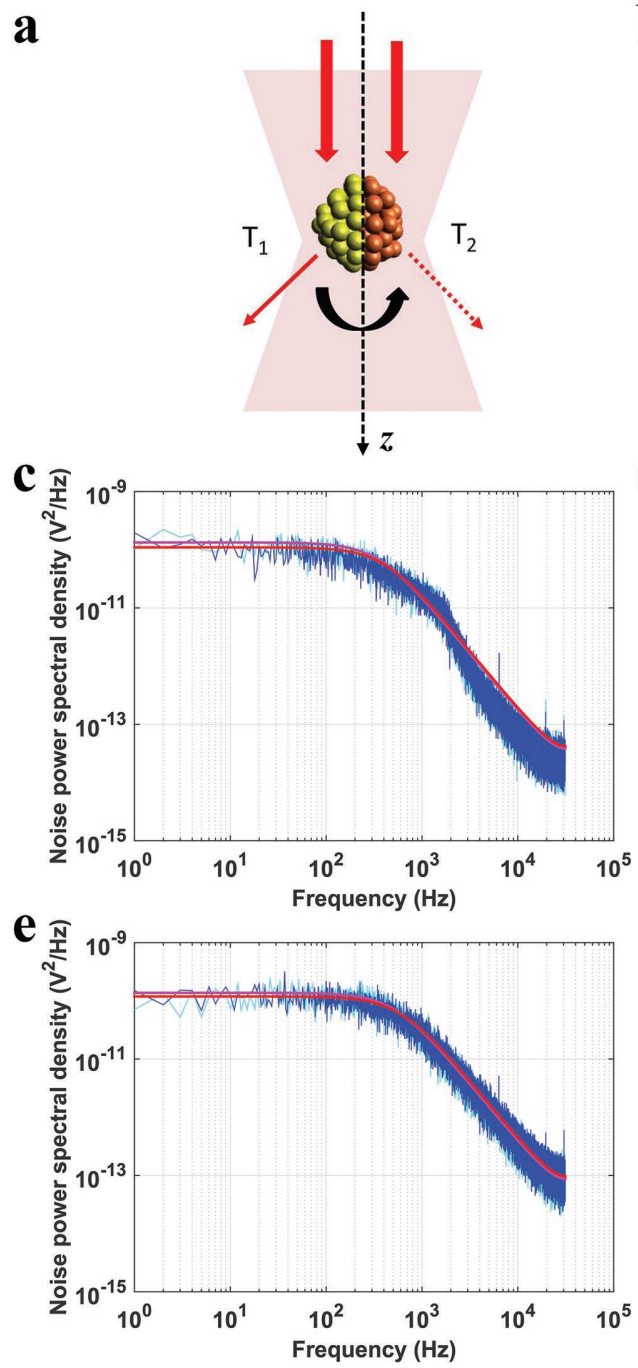

b

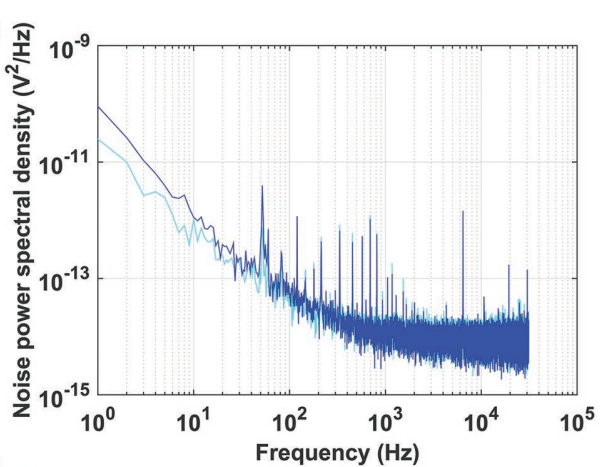

d

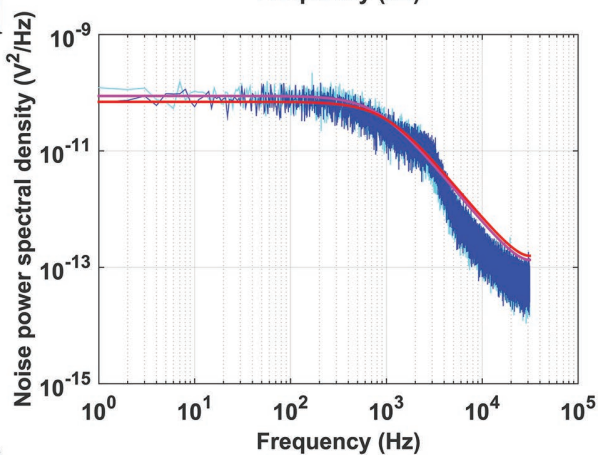

f



Figure 6. JNS displays colored Brownian motions in a single-beam gradient optical trap. a) A hypothetical cartoon to illustrate the local temperature imbalance around a single JNS particle, which drives the rotation of the particle at the focal plane. b) Power spectra for laser-only collected along $x$ - and $y$-axes. c,d) Representative power spectra for JNS particles displaying rotational motion in the optical trap. The best Lorentzian fits for each spectrum are overlaid, resulting in reduced $\chi^{2}$ values of 1.31 (red in panel (c)), 1.33 (purple in panel (c)), 1.21 (red in panel (d)), and 1.20 (purple in panel (d)), respectively. e,f) Representative power spectra for AuTCMs and FeTCMs in the optical trap, respectively. The best Lorentzian fits for each spectrum are overlaid, resulting in reduced $\chi^{2}$ values of 1.11 (red in (e)), 1.11 (purple in (e)), 1.10 (red in (f)) and 1.11 (purple in (f)), respectively. For panels $(b-f)$, the power spectra along the $x$-axis are shown in cyan while the power spectra along $y$-axis are shown in blue. The fits for spectra along $x$-axis are shown in red while the fits for spectra along $y$-axis are shown in purple. The $x y$ plane is the focal plane that is perpendicular to beam axis $z$.

(Figure 6b). Our data reveals that optically trapped JNS particles $(N=209)$ show a distinct peak at $0.5-3 \mathrm{kHz}$ in their power spectra (Figure 6c,d). The presence of these peaks, i.e., apparent "colors" in the thermal noise, ${ }^{[51]}$ is in sharp contrast to the power spectra displayed by homogeneous polystyrene or silica nanoparticles when they were trapped under identical conditions. ${ }^{[52]}$ As a consequence, a typical Lorentzian fitting for these power spectra yielded bad fits, as indicated by the systematic deviations between the power spectra and the best fitted curves overlaid on the spectra. As a matter of fact, these peaks are absent in all symmetric nanostructure controls including AuTCMs $(N=50)$ (Figure 6e) and FeTCMs $(N=75)$ (Figure 6f), respectively. For these power spectra, the Lorentzian fitting provides an adequate description for the data over the entire frequency range that we have investigated (1-30 kHz). Because these power spectra were recorded in the plane that is perpendicular to the laser beam axis ( $z$-axis in Figure 6a), the presence of the peaks along both $x$-and $y$-axes in this plane indicates that a population of JNS displays periodic rotational motions in the focal plane at a frequency between $0.5-3 \mathrm{kHz}$, which deviates from the typical Brownian motions, but is consistent with the rotation of these particles with a major frequency between 0.5 and $3 \mathrm{kHz}$ as driven by the local temperature gradient. The colors of thermal noise in Brownian motions has been demonstrated previously for microscopic particles under strong trapping conditions, ${ }^{[53]}$ which results from the correlated motions between a trapped particle and the surrounding solvent molecules, ${ }^{[54]}$ or the so-called hydrodynamic memory effects. The apparent colors in thermal noise that we observed here 
for the JNS particles must have a different origin. First of all, these JNSs are more than tenfold smaller in diameter than microspheres used by Franosch et al. ${ }^{[53]}$ Particles of this size are very weakly trapped, as we showed previously, ${ }^{[50,52]}$ and as a result, the effect of hydrodynamic memory is small. The absence of these peaks in the symmetric particles we used as a control further supports this notion, and argues that it is the asymmetry within the JNS particles that gives rise to this phenomenon.

While optical trapping of JNS has been previously demonstrated, those experiments were not designed to detect active motion. ${ }^{[25]}$ Our observation, however, is supported by work from Jiang et al. examining self-thermophoresis of gold/ silica Janus microparticles under $1064 \mathrm{~nm}$ laser irradiation, [24] It is worth noting that these asymmetrical JNS nanoparticles are more challenging to trap than symmetrical nanoparticles, due to the active spin motion. Although a portion of the current JNS sample exhibited a peak in the power spectra, the data are reproducible over multiple experiments. Moreover, the frequency for this periodic motion is also reasonable for a particle of this size in a single-beam gradient optical trap. ${ }^{[55]}$ Previous studies using polystyrene and gold nanoparticles subjected to circularly polarized light were able to achieve speeds up to $6 \mathrm{~Hz}$ and several $\mathrm{kHz}$, respectively, the latter at a laser power of $<50 \mathrm{~mW}$ and $\lambda=830 \mathrm{~nm} .{ }^{[56,57]}$ Furthermore, at these rotation speeds corresponding to high thermophoretic forces, it was predicted that the ability to optically trap JNS becomes strongly inhibited, leading to particle escape. ${ }^{[58]}$ This is a common occurrence during current trapping experiments, which suggests that a larger population of JNS may actually exhibit active rotational motion than what we were able to stably trap and measure here.

\section{Conclusion}

We have generated an asymmetrical multibuilding block JNS by employing a novel self-assembly approach that utilizes a thermocleavable polymer to control the location and selfassembly of nanoparticles. The data suggest that the asymmetrical JNS enhance photothermal effect and provide uneven thermophoretic force under NIR light, which generates active colored Brownian spin motion. These properties of the asymmetrical JNS could be used as both a strong photothermal mediator and a fuel-free nanoscale Janus motor under NIR light, which provides superior functions in the future compared to particles that have only either photothermal properties or rotational motion alone. JNS can simultaneously induce cancer cells death by increasing local temperature and potentially penetrate deeper in a tumor mass by the active rotational motion. These two properties make our JNS unique and possible to use in the future for cell manipulations, tissue penetration enhancement, and cargo deliveries. ${ }^{[59-61]}$

\section{Experimental Section}

Synthesis of Difurfuryl Adipate (DFA): DFA was synthesized using the previously published method. ${ }^{[62]}$ Briefly, $5.5 \mathrm{mmol}$ adipoyl chloride was added dropwise to $11 \mathrm{mmol}$ furfuryl alcohol with a few drops of triethanolamine (TEA) in dichloromethane (DCM). The reaction was carried out at $0{ }^{\circ} \mathrm{C}$ for $3 \mathrm{~h}$ under nitrogen atmosphere. The product was purified by silica gel column chromatography eluted with petroleum ether and ethyl acetate $(2: 1)$. The final product was brown viscous liquid. The chemical structure was confirmed by ${ }^{1} \mathrm{H}$ NMR spectroscopy (Varian $400 \mathrm{MHz}$ ) in DCM- $d_{4}$ and tetrachloro ethane (TCE)- $d_{2}$.

Synthesis of Cleavable Hydrophobic Backbone Polymer, DA Polymer: The DA polymer was synthesized from DFA and bismaleimido diphenyl methane (BMD) monomers following previous literature. ${ }^{[63,64]}$ An equimolar of DFA and BMD was mixed in TCE and the reaction was carried out at $70{ }^{\circ} \mathrm{C}$ for $7 \mathrm{~d}$. The viscous yellow liquid was precipitated by excess petroleum ether and pale yellow powder was obtained. The powder was dried out under vacuum conditions. The final product was characterized by ${ }^{1} \mathrm{HNMR}$ spectroscopy in TCE- $d_{2}$. Molecular weight of DA was measured by GPC.

Cycloadduct Conversion: In order to determine the percentage of cycloadduct conversion, DFA and BMD were reacted at $70{ }^{\circ} \mathrm{C}$ for $48 \mathrm{~h}$ to induce DA reaction. Retro DA occurred at $100^{\circ} \mathrm{C}$ and led to the cycloadduct cleavage. The percentage of cycloadduct conversion was calculated from the area under the peaks appearing in ${ }^{1} \mathrm{H}$ NMR at 5.32 and 7.43 ppm, respectively. They indicated cycloadducts in the hydrophobic backbone and furan rings in the starting material respectively

\%Conversion

$$
=\left\{\frac{\mathrm{AUC} \text { at } 5.32 \mathrm{ppm}}{(\mathrm{AUC} \text { at } 5.32 \mathrm{ppm}+\mathrm{AUC} \text { at } 7.43 \mathrm{ppm})}\right\} \times 100
$$

Synthesis of Thermocleavable Polymer (DA-b-PEO) via Michael Addition: The excess molar concentration of thiol-methoxy polyethylene oxide, molecular weight $5000 \mathrm{Da}$ (SH-mPEO), was added into the solution of DA polymer (1.5:1 molar ratio) in DCM with a few drops of TEA. The solution was kept under stirring for one night and the product was precipitated into petroleum ether. The polymer structure was confirmed by ${ }^{1} \mathrm{H}$ NMR spectroscopy. Molecular weight was determined by GPC.

Synthesis of IONPS: $15 \mathrm{~nm}$ IONPs were synthesized using a previously reported method. ${ }^{[65]}$ Briefly, a mixture of $0.890 \mathrm{~g}$ $\mathrm{FeO}(\mathrm{OH}), 19.8 \mathrm{~g}$ oleic acid, and $25.0 \mathrm{~g} 1$-octadecene in a threeneck flask was heated under stirring to $200{ }^{\circ} \mathrm{C}$ under $\mathrm{N}_{2} .30 \mathrm{~min}$ later the temperature was set at $220^{\circ} \mathrm{C}$ for $1 \mathrm{~h}$, then the temperature was increased gradually to $310^{\circ} \mathrm{C}\left(20^{\circ} \mathrm{C} / 5 \mathrm{~min}\right)$ and kept at this temperature for $1 \mathrm{~h}$. The solution became black when the temperature was increased to $320^{\circ} \mathrm{C}$ and kept at this temperature for $1 \mathrm{~h}$. After the reaction was completed, the reaction mixture was cooled and the nanocrystals were precipitated by adding chloroform and acetone.

Au/IONP JNS Formation: FeTCM $\left(2.7 \times 10^{-9} \mathrm{M}\right)$, AuTCM $\left(0.2 \times 10^{-6} \mathrm{M}\right)$, and DA-b-PEO $\left(10.0 \times 10^{-6} \mathrm{M}\right)$ were mixed together at room temperature. The solution was subsequently heated at $94^{\circ} \mathrm{C}$ for $3 \mathrm{~h}$ in a dry bath. The obtained JNSs were centrifuged twice to remove free polymer seeds. The JNS solution was kept at $4{ }^{\circ} \mathrm{C}$ in the magnet separator (EasySep, USA) for one night to remove unreacted AuTCM.

Synthesis of FeBL and AUPS Secondary Nanostructures: To form iron oxide ball-like nanostructures $(\mathrm{FeBL})$, FeTCM $\left(0.54 \times 10^{-9} \mathrm{M}\right)$ 
were homogeneously mixed with TCM seeds $\left(0.2 \times 10^{-6} \mathrm{M}\right)$ at room temperature. The formation of the ball-like structures was carried out at $95^{\circ} \mathrm{C}$ for $2 \mathrm{~h}$ in a dry bath. Then the solution was centrifuged to remove free TCM seeds and the pallets of ball-like nanostructures were redispersed in Milli $Q$ water. To form gold patchy nanostructures (AuPS), AuTCM $\left(0.4 \times 10^{-6} \mathrm{~m}\right)$ was mixed with free TCM seeds $\left(10.0 \times 10^{-6} \mathrm{M}\right)$. The solution was heated at $94^{\circ} \mathrm{C}$ for $3 \mathrm{~h}$ in a heat block and purified by centrifugation. The final products were stored at $4^{\circ} \mathrm{C}$.

Synthesis of Homogeneous Au/IONP Micelles (GMC): Oleic acid coated-IONPs (1 mg) and dodecanethiol AuNPs (1 mg) were dissolved together in THF and subsequently added into $20 \mathrm{mg}$ of DA-b-PEO solution in tetrahydrofuran (THF) under stirring. The resulting solution was slowly dropped into water under vigorous agitation. The product was purified twice by weight-separated centrifugation.

Characterization of Nanoparticle: NP-TCM, secondary structures, and JNS samples for TEM imaging were prepared by the solvent evaporation method. Briefly, the solution $(5 \mu \mathrm{L})$ of each sample were dropped onto carbon-coated copper TEM grids and allowed to dry overnight. TEM images were acquired on a transmission electron microscope (TEM, Phillips CM-100, 60 kV). STEM and XEDS were performed using Jeol JEM-2010F, operating at $200 \mathrm{kV}$ with a double tilt holder. Images and size distributions were analyzed by ImageJ software from NIH and Gatan digital image software. Hydrodynamic diameters were measured by using Malvern Zeta Sizer Nano S-90. Concentrations of Fe and Au were quantified by Inductively Coupled Plasma-Optical Emission Spectroscopy (Perkin-Elmer Optima 2000 DV). The samples were digested in aqua regia overnight before measurement. Percent yield of JNS was calculated by Equation (2) shown below

$\%$ yield of JNS $=\frac{(\text { Amount of Fe in JNS } \times 100)}{\text { Amount of Fe in FeTCM }}$

Photothermal Effect and Cell Viability Assay in Triple Negative Breast Cancer Cells (SUM-159): The cells were cultured in a $5 \% \mathrm{CO}_{2}$ environment in $\mathrm{F} 12$ media (Invitrogen, Carlsbad, CA) supplemented with $5 \%$ fetal bovine serum (Fisher Scientific, Pittsburgh, PA), 1\% antibiotic-antimycotic (Invitrogen, Carlsbad, (A), $5 \mu \mathrm{g} \mathrm{mL} \mathrm{m}^{-1}$ insulin (Sigma-Aldrich, St Louis, MO), $1 \mu \mathrm{g} \mathrm{mL}$ hydrocortisone (Sigma-Aldrich, St Louis, MO), and $4 \mu \mathrm{g} \mathrm{mL}$ gentamicin (Invitrogen, Carlsbad, CA). $3 \times 10^{3}$ cells were seeded in 96-well plates overnight. The cells were incubated with JNS $\left(0.17 \mathrm{mg} \mathrm{mL}^{-1}\right.$ of gold and $0.04 \mathrm{mg} \mathrm{mL}^{-1}$ of iron), AuTCM (0.17 mg mL $\mathrm{mb}^{-1}$ of gold), FeTCM (0.04 $\mathrm{mg} \mathrm{mL}^{-1}$ of iron), GMC (0.17 $\mathrm{mg} \mathrm{mL}^{-1}$ of gold and $0.03 \mathrm{mg} \mathrm{mL}^{-1}$ of iron), solution mixture of AuTCM $\left(0.17 \mathrm{mg} \mathrm{mL}^{-1}\right.$ of gold) and FeTCM $\left(0.04 \mathrm{mg} \mathrm{mL}^{-1}\right.$ of iron), or cell culture media alone for $2 \mathrm{~h}$. The cells were irradiated using an NIR laser $\left(885 \mathrm{~nm}, 0.45 \mathrm{~W}\right.$, spot size $5 \times 8 \mathrm{~mm}^{2}$, MDL-III-885, OPTO Engine LLC, Midvale, UT) for $10 \mathrm{~min}$. The temperature of the solutions was measured using a thermal camera (FLIR, Boston, MA). Nanoparticles were removed immediately after PTT and the cells were incubated overnight. Alamar blue (BIO-RAD, Raleigh, NC) was added to each well and incubated for $4 \mathrm{~h}$ before fluorescent cell viability measurement at Excitation: $540 \mathrm{~nm}$ and Emission: $590 \mathrm{~nm}$.

Optical Trapping of JNS: A custom-built optical tweezers instrument incorporating a tapered amplifier diode laser at $830 \mathrm{~nm}$ (SYS-420-830-1000, Sacher LaserTechnik LLC, Germany) was used for trapping AuTCM, FeTCM, and JNS samples. Briefly, the trapping laser was focused to a diffraction-limited spot using a 60X waterimmersion objective (Nikon, Melville, NY) with a numerical aperture of 1.2. The laser power was $130.8 \mathrm{~mW}$ at focus throughout the experiments, which were conducted at $20 \pm 0.2{ }^{\circ} \mathrm{C}$. Samples were diluted tenfold in distilled water and separately injected into a microfluidic chamber of our construction. The optical trap was initially stationary, allowing individual, diffusing nanoparticles to be passively captured. Brownian motion of trapped particles was recorded with BFPI at $62.5 \mathrm{kHz}$ for $10 \mathrm{~s}$ for the two axes that were perpendicular to the $z$-axis, i.e., the laser beam axis along the direction of beam propagation. This scan was repeated four times. Fitting of the corresponding power spectra in MATLAB (The Mathworks, Natick, MA) was done as previously described. ${ }^{[50]}$ If the particle still remained in the optical trap after all scans were completed, it was released and a new particle was trapped after sufficient time had elapsed.

\section{Supporting Information}

Supporting Information is available from the Wiley Online Library or from the author.

\section{Acknowledgements}

The authors gratefully acknowledge scholarship support provided to K.S. by the Thai Government Scholarship Council. The authors thank Microscopy \& Image Analysis Laboratory (MIL), The Michigan Center for Materials Characterization for imaging facilities and NSF grant \#DMR-9871177. The authors thank Marisa Gies for her assistance with the preparation of this manuscript.

[1] C. Kaewsaneha, P. Tangboriboonrat, D. Polpanich, M. Eissa, A. Elaissari, ACS Appl. Mater. Interfaces 2013, 5, 1857.

[2] A. Perro, S. Reculusa, S. Ravaine, E. Bourgeat-Lami, E. Duguet, J. Mater. Chem. 2005, 15, 3745.

[3] L.-T.-C. Tran, S. Lesieur, V. Faivre, Expert Opin. Drug Delivery 2014, $11,1061$.

[4] S. Bhaskar, C. T. Gibson, M. Yoshida, H. Nandivada, X. Deng, N. H. Voelcker, J. Lahann, Small 2011, 7, 812.

[5] J. Du, R. K. O’Reilly, Chem. Soc. Rev. 2011, 40, 2402.

[6] S.-H. Hu, X. Gao, J. Am. Chem. Soc. 2010, 132, 7234.

[7] W. Gao, A. Pei, X. Feng, C. Hennessy, J. Wang, J. Am. Chem. Soc. 2013, 135, 998.

[8] J. Reguera, H. Kim, F. Stellacci, Chimia 2013, 67, 811.

[9] S. K. Ghosh, T. Pal, Chem. Rev. 2007, 107, 4797.

[10] L. Yan, N. Popp, S. Ghosh, A. Boker, ACS Nano 2010, 4, 913.

[11] S. Jiang, Q. Chen, M. Tripathy, E. Luijten, K. S. Schweizer, S. Granick, Adv. Mater. 2010, 22, 1060.

[12] M. Lattuada, T. A. Hatton, Nano Today 2011, 6, 286.

[13] M. Lattuada, T. A. Hatton, J. Am. Chem. Soc. 2007, 129, 12878.

[14] F. Wurm, A. F. M. Kilbinger, Angew. Chem., Int. Ed. Engl. 2009, 48, 8412.

[15] R. J. Hickey, J. Koski, X. Meng, R. a Riggleman, P. Zhang, S.-J. Park, ACS Nano 2014, 8, 495.

[16] S. S. Lamarre, C. Lemay, C. Labrecque, A. M. Ritcey, Langmuir 2013, 29, 10891. 
[17] J. He, X. Huang, Y.-C. Li, Y. Liu, T. Babu, M. a Aronova, S. Wang, Z. Lu, X. Chen, Z. Nie, J. Am. Chem. Soc. 2013, 135, 7974.

[18] M. Kanahara, M. Shimomura, H. Yabu, Soft Matter 2014, 10, 275.

[19] R. J. Hickey, X. Meng, P. Zhang, S.-J. Park, ACS Nano 2013, 7, 5824.

[20] E. R. Zubarev, J. Xu, A. Sayyad, J. D. Gibson, J. Am. Chem. Soc. 2006, 128, 15098.

[21] Z. Zhang, M. A. Horsch, M. H. Lamm, S. C. Glotzer, Nano Lett. 2003, 3, 1341.

[22] X. Huang, M. A. El-Sayed, J. Adv. Res. 2010, 1, 13.

[23] S. Shen, S. Wang, R. Zheng, X. Zhu, X. Jiang, D. Fu, W. Yang, Biomaterials 2015, 39, 67.

[24] H. R. Jiang, N. Yoshinaga, M. Sano, Phys. Rev. Lett. 2010, $105,1$.

[25] S. Nedev, S. Carretero-Palacios, P. Kühler, T. Lohmüller, A. S. Urban, L. J. E. Anderson, J. Feldmann, ACS Photonics 2015, 2, 491.

[26] Z. Wu, X. Lin, T. Si, Q. He, Small 2016, 12, 3080.

[27] Y. Wu, Z. Wu, X. Lin, Q. He, J. Li, ACS Nano 2012, 6, 10910.

[28] Z. Wu, X. Lin, Y. Wu, T. Si, J. Sun, Q. He, ACS Nano 2014, 8, 6097.

[29] Z. Wu, X. Lin, T. Si, Q. He, Small 2016, 12, 3080.

[30] C. Goussé, A. Gandini, Polym. Int. 1999, 48, 723.

[31] A. Gandini, Prog. Polym. Sci. 2013, 38, 1.

[32] D. J. Hall, H. M. Van Den Berghe, A. P. Dove, Polym. Int. 2011, 60, 1149.

[33] M. Grzelczak, J. Vermant, E. M. Furst, L. M. Liz-marza, ACS Nano 2010, 4, 3591.

[34] L. Luo, A. Eisenberg, Langmuir 2001, 17, 6804.

[35] S. C. Owen, D. P. Y. Chan, M. S. Shoichet, Nano Today 2012, 7, 53.

[36] H. Deng, F. Dai, G. Ma, X. Zhang, Adv. Mater. 2015, 27, 3645.

[37] H. Deng, Y. Zhong, M. Du, Q. Liu, Z. Fan, F. Dai, X. Zhang, Theranostics 2014, 4, 904.

[38] Y. Liu, Y. Li, J. He, K. J. Duelge, Z. Lu, Z. Nie, J. Am. Chem. Soc. 2014, 136, 2602.

[39] M. S. Nikolic, C. Olsson, A. Saldier, A. Kornowski, A. Rank, R. Schubert, A. Frömsdorf, H. Weller, S. Förster, Angew. Chem., Int. Ed. 2009, 48, 2752.

[40] M. E. Mackay, A. Tuteja, P. M. Duxbury, C. J. Hawker, B. Van Horn, Z. Guan, G. Chen, R. S. Krishnan, Science 2006, 311, 1740.

[41] R. B. Thompson, V. V. Ginzburg, M. W. Matsen, A. C. Balazs, Science 2001, 292, 2469.

[42] A. Walther, K. Matussek, A. H. E. Muller, ACS Nano 2008, 2, 1167.
[43] D. Jaque, L. Martínez Maestro, B. del Rosal, P. Haro-Gonzalez, A. Benayas, J. L. Plaza, E. Martín Rodríguez, J. García Solé, Nanoscale 2014, 6, 9494.

[44] J. R. Melamed, R. S. Edelstein, E. S. Day, ACS Nano 2015, 9, 6.

[45] X. Huang, M. A. El-Sayed, Alexandria J. Med. 2011, 47, 1.

[46] X. Huang, I. H. El-Sayed, W. Qian, M. A. El-Sayed, J. Am. Chem. Soc. 2006, 128, 2115.

[47] A. O. Govorov, H. H. Richardson, Nano Today 2007, 2, 30.

[48] A. O. Govorov, W. Zhang, T. Skeini, H. Richardson, J. Lee, N. A. Kotov, Nanoscale Res. Lett. 2006, 1, 84.

[49] W. Zhang, Q. Li, M. Qiu, Opt. Express 2013, 21, 172.

[50] Y. Pang, H. Song, J. H. Kim, X. Hou, W. Cheng, Nat. Nanotechnol. 2014, 9, 624.

[51] P. Hänggi, P. Jung, Adv. Chem. Phys. 1994, 89, 239.

[52] Y. Pang, H. Song, W. Cheng, Biomed. Opt. Express 2016, 7, 1672.

[53] T. Franosch, M. Grimm, M. Belushkin, F. Mor, G. Foffi, L. Forró, S. Jeney, Nature 2011, 478, 8.

[54] K. Berg-Sorensen, H. Flyvbjerg, New J. Phys. 2005, 7, 38.

[55] M. E. J. Friese, T. A. Nieminen, N. R. Heckenberg, H. Rubinsztein-Dunlop, Nature 1998, 394, 348.

[56] K. Wang, E. Schonbrun, P. Steinvurzel, K. B. Crozier, Nat. Commun. 2011, 2, 469.

[57] A. Lehmuskero, R. Ogier, T. Gschneidtner, P. Johansson, M. Käll, Nano Lett. 2013, 13, 3129.

[58] R. T. Schermer, C. C. Olson, J. P. Coleman, F. Bucholtz, Opt. Express 2011, 19, 10571.

[59] W. Gao, R. Dong, S. Thamphiwatana, J. Li, W. Gao, L. Zhang, J. Wang, ACS Nano 2014, 9, 117.

[60] L. Baraban, D. Makarov, R. Streubel, I. Mönch, D. Grimm, S. Sanchez, O. G. Schmidt, ACS Nano 2012, 6, 3383.

[61] L. Baraban, M. Tasinkevych, M. N. Popescu, S. Sanchez, S. Dietrich, O. G. Schmidt, Soft Matter 2012, 8, 48.

[62] N. Kuramoto, K. Hayashi, K. Nagai, J. Polym. Sci., Part A: Polym. Chem. 1994, 32, 2501.

[63] A. Gandini, D. Coelho, M. Gomes, B. Reis, A. Silvestre, J. Mater. Chem. 2009, 19, 8656.

[64] A. Gandini, D. Coelho, A. J. D. Silvestre, Eur. Polym. J. 2008, 44, 4029.

[65] H. Chen, H. Paholak, M. Ito, K. Sansanaphongpricha, W. Qian, Y. Che, D. Sun, Nanotechnology 2013, 24, 355101.

Received: August 2, 2016

Revised: September 16, 2016

Published online: November 22, 2016 